\section{Influência da obesidade na força muscular de membros inferiores e superiores em adolescentes}

\section{Influence of obesity on the upper and lower body muscular strength in adolescents}

Wendell Arthur Lopes ${ }^{1,2}$

Neiva Leite ${ }^{2}$

Larissa Rosa da Silva ${ }^{2}$

Frederico Bento de Moraes Junior ${ }^{2}$

Cássio Leandro Mühe Consentino ${ }^{2}$

Cristiane Tavares Araújoº

Claudia Regina Cavaglieri ${ }^{1}$

\section{Resumo}

Comparar a força muscular de membros inferiores e superiores entre adolescentes obesas e não-obesas e verificar a associação da força muscular com as variáveis antropométricas e da composição corporal. Participaram 36 adolescentes, do sexo feminino, com idade entre 13-17 anos, divididas em três grupos: obeso $(n=13)$, sobrepeso $(n=13)$ e eutrófico $(n=10)$. Foram medidos massa corporal, estatura, índice de massa corporal (IMC) e a circunferência da cintura. A composição corporal foi mensurada pelo método de impedância bioelétrica e a força muscular estimada pelo teste de uma repetição máxima. As adolescentes obesas apresentaram maiores valores de força muscular absoluta nos membros inferiores $(F=10,43 ; p=0,0003)$ e nos superiores $(\mathrm{F}=4,72 ; \mathrm{p}=0,01)$ que as não-obesas. $\mathrm{O} \mathrm{IMC}$, a massa gorda $(\mathrm{MG})$ e a massa livre de gordura (MLG) foram direta e significativamente correlacionados com a força muscular de membros inferiores $(r=0,66, p<0,01 ; r=0,58, p<0,01 ; r=0,54, p<0,01)$ e superiores $(r=0,54, p<0,01 ; r=$ $0,43, p<0,01 ; r=0,57, p<0,01)$, respectivamente. A regressão linear mostrou que o IMC explicou $44 \%$ da variação da força muscular absoluta dos membros inferiores $(\beta=4,81, p<0,01)$ e a MLG explicou $32 \%$ da variação da força muscular absoluta dos membros superiores $(\beta=0,52, p<0,05)$. A obesidade afetou a força muscular absoluta de membros inferiores e superiores em adolescentes e a magnitude deste efeito foi maior nos membros inferiores. O IMC foi a variável que melhor explicou a variação da força muscular absoluta dos membros inferiores em adolescentes obesas e não-obesas na amostra estudada.

\section{Palavras-chave}

Obesidade, Força muscular, Adolescente, Composição corporal, Aptidão física.

\begin{abstract}
To compare the muscle strength of upper and lower limbs among adolescent obese and non-obese and the association of muscle strength with anthropometric and body composition. Thirty-six adolescent girls, with 13-17 years-old, participated in the study and were divided into three groups: obese ( $n=$ $13)$, overweight $(n=13)$ and healthy $(n=10)$. Body mass, height, body mass index (BMI) and waist circumference were evaluated. Body composition was measured by the bioelectric impedance method. Muscular strength was estimated by 1RM. Obese girls showed higher absolute muscle strength in the lower $(F=10.43, p=0.0003)$ and higher $(F=4.72, p=0.01)$ than non-obese. The BMI, fat mass (FM) and fat free mass (FFM) were directly and significantly correlated with lower limb muscle strength $(r=0.66, p<0.01 ; r=0.58, p<0.01 ; r=0.54, p<0.01)$ and higher $(r=0.54, p<0.01 ; r=0$, $43, p<0.01 ; r=0.57, p<0.01)$, respectively. Linear regression showed that BMI explained $44 \%$ of the variation in absolute muscle strength of the lower limbs $(\beta=4.81, p<0.01)$ and FFM explained $32 \%$ of the variation in strength absolute muscle of the upper $(\beta=0.52, p<.05)$. Obesity affected absolute muscle strength of the lower limbs and in the upper teens and the magnitude of the effect was greater in the lower limbs than in the upper. BMI was the variable that best explained the variation in absolute muscle strength of the lower limbs in adolescent obese and non-obese in the assessed sample.
\end{abstract}

\section{Keywords}

Obesity, Strength muscle, Adolescent, Body composition, Physical fitness.
Rev Bras Ativ Fis Saúde p. 720-729 DOl:

http://dx.doi.org/10.12820/rbafs.v.18n6p720

1 Laboratório de Fisiologia do Exercício (FISEX), Faculdade de Educação Física, Universidade Estadual de Campinas (UNICAMP), Campinas, São Paulo

2 Núcleo de Pesquisa em Qualidade de Vida (NQV), Departamento de Educação Física, Universidade Federal do Paraná (UFPR), Curitiba, Paraná 


\section{INTRODUÇÃO}

A obesidade aumentou entre crianças e adolescentes nas últimas décadas ${ }^{1}$, principalmente em países em desenvolvimento ${ }^{2}$. No Brasil, a prevalência de sobrepeso e obesidade aumentou de 4,1 para 13,9\% durante 1974-19972. O excesso de peso nessa fase da vida tem sido associada a doenças cardiovasculares, pulmonares, diabetes e distúrbios psicossociais, bem como maior risco de desenvolvimento de doenças na vida adulta ${ }^{3}$. Além disto, o excesso de peso está relacionado com baixos níveis de atividade física e redução da aptidão física ${ }^{4}$. De fato, indivíduos obesos apresentam menor aptidão cardiorrespiratória, representado por menores valores de consumo máximo de oxigênio $\left(\mathrm{VO}_{2 \mathrm{max}}\right)$, quando expressado de forma relativa à massa corporal ${ }^{5}$.

A força muscular tem sido reconhecida como componente importante para a saúde $^{6}$. Em adultos, menores níveis de força muscular têm sido associados com maior risco de mortalidade por todas as causas ${ }^{7}$. Em crianças e adolescentes, parece que elevados níveis de força muscular estão inversa e positivamente associados com risco metabólico ${ }^{8}$ e resistência insulínica ${ }^{9,10}$. No entanto, o impacto da obesidade na funcionalidade musculoesquelética tem sido pouco investigado ${ }^{5}$.

Estudos que analisaram a força muscular entre obesos e não obesos têm encontrado valores similares ou maiores de força absoluta que seus pares não obesos ${ }^{11-19}$. Esses achados têm sido atribuídos a provável adaptação neuromuscular induzida pelo excesso de peso corporal na estrutura musculoesquelética. Por outro lado, quando a força muscular é expressa por unidade de massa corporal ou de massa livre de gordura essas diferenças desapareceram, sugerindo que a qualidade do tecido muscular não é afetada pela obesidade ${ }^{19}$. É interessante notar que a maioria desses estudos verificou a força muscular dos membros inferiores, os quais são mais influenciados pelo excesso de peso corporal, dificultando assim a identificação dos prováveis efeitos da obesidade na expressão da força muscular. Somente um estudo investigou a força muscular de membros superiores em mulheres obesas ${ }^{15}$, o qual mostrou maiores valores de força muscular absoluta em comparação as não-obesas, sugerindo que os membros superiores também são afetados pela obesidade. Até o presente momento, nenhum estudo investigou o efeito da obesidade na força muscular dos membros superiores em adolescentes, a fim de verificar se os efeitos da obesidade na força muscular já estão presentes nessa faixa etária. Além disto, nenhum estudo verificou se a obesidade afeta diferentemente os membros inferiores e superiores de indivíduos obesos em relação aos não-obesos. Portanto, o propósito do presente estudo foi: a) comparar a força muscular de membros superiores e inferiores entre adolescentes obesas e não-obesas; b) verificar a associação entre as variáveis antropométricas e de composição corporal e a força muscular dos membros inferiores e superiores em adolescentes.

\section{MÉTODOS}

Estudo transversal composto por 36 adolescentes do sexo feminino, com idade entre 13-17 anos, selecionados por conveniência e provenientes de escola pública de Curitiba/Paraná. Os sujeitos foram alocados em três grupos: obeso $(n=13)$, sobrepeso $(n=13)$ e eutrófico $(n=10)$, conforme os pontos de corte para classificação do estado nutricional proposto pela Organização Mundial da Saúde ${ }^{20}$. Os participantes não apresentavam nenhuma contraindicação para realização dos exames, incluindo ausência de doenças cardíacas, pulmonares e osteoarticulares 
que comprometessem a realização dos testes de força muscular. Os voluntários não realizavam outra atividade física regular, nos últimos seis meses, além da educação física escolar (120 min/sem). Todos os voluntários e os pais ou responsáveis foram informados e assinaram o termo de consentimento livre e esclarecido, conforme projeto de pesquisa aprovado pelo Comitê de Ética em Pesquisa em Seres Humanos (protocolo no 2460.067/2011-03).

A massa corporal $(\mathrm{MC})[\mathrm{kg}]$ foi aferida em balança Filizola ${ }^{\circledR}$, com precisão de $0,1 \mathrm{~kg}$, tipo plataforma, com o avaliado permanecendo em posição ortostática, descalço, pés unidos no centro da plataforma e vestindo roupas leves. A estatura (cm) foi aferida em estadiômetro fixado à balança, com precisão de $0,1 \mathrm{~cm}$, estando o indivíduo descalço, pés unidos no centro da plataforma, cabeça posicionada no plano horizontal de Frankfurt ao final de inspiração máxima. A circunferência cintura (CC) foi realizada utilizando fita antropométrica inextensível com resolução de $0,1 \mathrm{~cm}$ sendo a medida obtida no ponto médio entre o último arco costal e a crista ilíaca. Foram realizadas três medidas no mesmo local, sendo considerado o valor mediano entre elas.

Para avaliação da composição corporal foi adotado o método de impedância bioelétrica (BIA) tetrapolar, utilizando aparelho Maltron ${ }^{\circledR}$, modelo BF906, com frequência de $50 \mathrm{khz}$. O procedimento foi realizado no período da manhã, após jejum de 10-12 horas, em decúbito dorsal. Os eletrodos foram posicionados nas superfícies dorsais das mãos e dos pés direitos, próximo às articulações metacarpo-falangeanas e metatarso-falangeanas e medialmente entre as proeminências distais do rádio e da ulna, entre o maléolo tibial e fibular. Obteve-se a resistência e calculou-se a massa livre de gordura (MLG) por meio da equação proposta por Houtkooper ${ }^{21}$, disponível no programa do equipamento.

A avaliação puberal foi realizada por inspeção visual, realizada por médico pediatra experiente com base no estadiamento proposto por Tanner ${ }^{22}$, classificando o desenvolvimento mamário (M1-M5) e pilificação pubiana (P1-P5).

A força muscular foi estimada com base na carga movimenta obtida em teste de uma repetição máxima (1RM), conduzido conforme protocolo proposto por Brown \& Weir ${ }^{23}$. Os grupos compareceram três vezes (3 visitas) ao laboratório para realização do teste, sendo uma familiarização aos equipamentos e procedimentos e 2 visitas para a realização do teste de 1 RM. Os exercícios utilizados foram o Bench Press (BP) e Leg Press (LP), sendo realizado nessa ordem em todas as visitas. Com o objetivo de evitar influências circadianas na força muscular todas as visitas foram realizadas no período da tarde (14-16 horas). Os testes foram distribuídos em 3 dias não consecutivos, com intervalo de 72 horas, para evitar os efeitos residuais das outras sessões no teste de 1RM. A fim de evitar influências interavaliador, os testes foram conduzidos por único avaliador experiente. Antecedendo ao início de cada sessão, os sujeitos realizaram aquecimento de 5 minutos de caminhada em esteira ergométrica, com velocidade entre 4 e $5 \mathrm{~km} / \mathrm{h}$. Na primeira visita foi realizada a familiarização aos exercícios que compuseram o teste de $1 \mathrm{RM}$, para essa fase foi realizado 3 séries de 8 a 10 repetições com cargas progressivas afim de atingir aproximadamente $50 \%$ da carga com base na sua percepção subjetiva do esforço. A segunda visita, iniciou-se com aquecimento localizado com uma série de 8 repetições a aproximadamente $50 \%$ da carga percebida na primeira visita. Após 1 minuto de intervalo foi realizada a segunda série de 3 repetições a $70 \%$ da carga percebida, após 2 minutos de intervalo os sujeitos foram submetidos ao primeiro teste de 1RM com 100\% (considerando o dobro da carga percebida na primeira visita 50\%) da carga percebida, após a execução do 1RM foi realizado intervalo de 
3 à 5 minutos para correção da carga. Quando o $1 \mathrm{RM}$ foi realizado com sucesso, foram acrescidos de $5 \%$ a $10 \%$ da carga até se alcançar a carga máxima para uma repetição realizada corretamente. Quando houve o insucesso no 1RM foi realizado decréscimo da carga em $50 \%$ do incremento realizado na ultima tentativa. $\mathrm{Na}$ terceira visita foi realizado o teste de $1 \mathrm{RM}$ seguindo os mesmos procedimentos descritos na segunda visita, porém utilizando as cargas alcançadas na segunda visita para fins de cálculos dos percentuais utilizados no aquecimento e no teste propriamente dito. Nesta visita o objetivo foi confirmar a carga de $1 \mathrm{RM}$ da sessão anterior ou realizar novos incrementos de carga quando necessários. Considerouse a maior carga alcançada na última visita como valor de $1 \mathrm{RM}$ para o $\mathrm{BP}$ e para o LG. A força muscular estimada pelo teste de $1 \mathrm{RM}$ foi expressa em termos absolutos (carga absoluta movimentada), relativo à $\mathrm{MC}$ (carga movimentada dividida pela $\mathrm{MC}$ ) e relativo à MLG (carga movimentada dividida pela MLG).

Os dados foram testados quanto à normalidade e homogeneidade de variâncias pelos testes de Shapiro-Wilk e Levene, respectivamente. Para a comparação das variáveis antropométricas, composição corporal e força muscular foi utilizada a ANOVA one-way seguido do teste comparações múltiplas de Tukey. O teste QuiQuadrado $\left(\chi^{2}\right)$ foi adotado para as comparações das proporções nos diferentes estágios da maturação sexual entre os grupos. O coeficiente de correlação produtomomento de Pearson foi utilizado para verificar a correlação entre a força muscular absoluta (membros inferiores e superiores) e as variáveis antropométricas (IMC) e de composição corporal (\%GC, MG e MLG). A regressão linear múltipla foi utilizada para verificar o grau de associação entre as variáveis antropométricas e da composição corporal (variáveis independentes) e a força muscular absoluta dos membros inferiores e superiores (desfecho). Foi utilizado o método de seleção stepwise, sendo o critério de entrada no modelo as variáveis com valor de $\mathrm{p}<0,20$. O nível de significância adotado foi $\mathrm{p}<0,05$ para teste bicaudal.

\section{RESULTADOS}

As características gerais dos participantes do estudo estão apresentadas na Tabela 1. Não houve diferença significativa na idade e na estatura entre os grupos. Os valores de MC, IMC e MG foram diferentes entre todos os grupos. A CC, o \%MG e a MLG foram maiores nos indivíduos obesos e sobrepesados em comparação aos eutróficos. O \%MLG foi menor entre os obesos em comparação aos não-obesos (sobrepeso e eutrófico).

Os valores da carga máxima movimentada no teste de 1RM nos membros inferiores (Leg Press) e superiores (Bench Press) em termos absoluto, relativos à MC e à MLG estão representados na Figura 1. Quando a carga movimentada foi expressa em termos absolutos, as obesas apresentaram maiores valores nos membros inferiores em comparação às não-obesas $(\mathrm{F}=10,43 ; \mathrm{p}=0,0003)$ e nos superiores em relação às eutróficas $(\mathrm{F}=4,72 ; \mathrm{p}=0,01)$ (Figura $1 \mathrm{~A}$ e $\mathrm{B})$. Por outro lado, quando a carga movimentada foi expressa em termos relativos à $\mathrm{MC}$, as obesas apresentaram menores valores nos membros superiores $(F=3,63 ; p=0,03)$ (Figura 1D) em comparação às eutróficas. E quando a carga movimentada foi expressa em termos relativos à MLG, não houve diferença estatística significativa entre nenhum dos grupos avaliados (Figura 1E e F).

A Tabela 2 apresenta os valores dos coeficientes de correlação de Pearson entre a carga movimentada nos membros inferiores e superiores e as variáveis antropométricas e da composição corporal. O IMC, MG e a MLG foram direta e signi- 

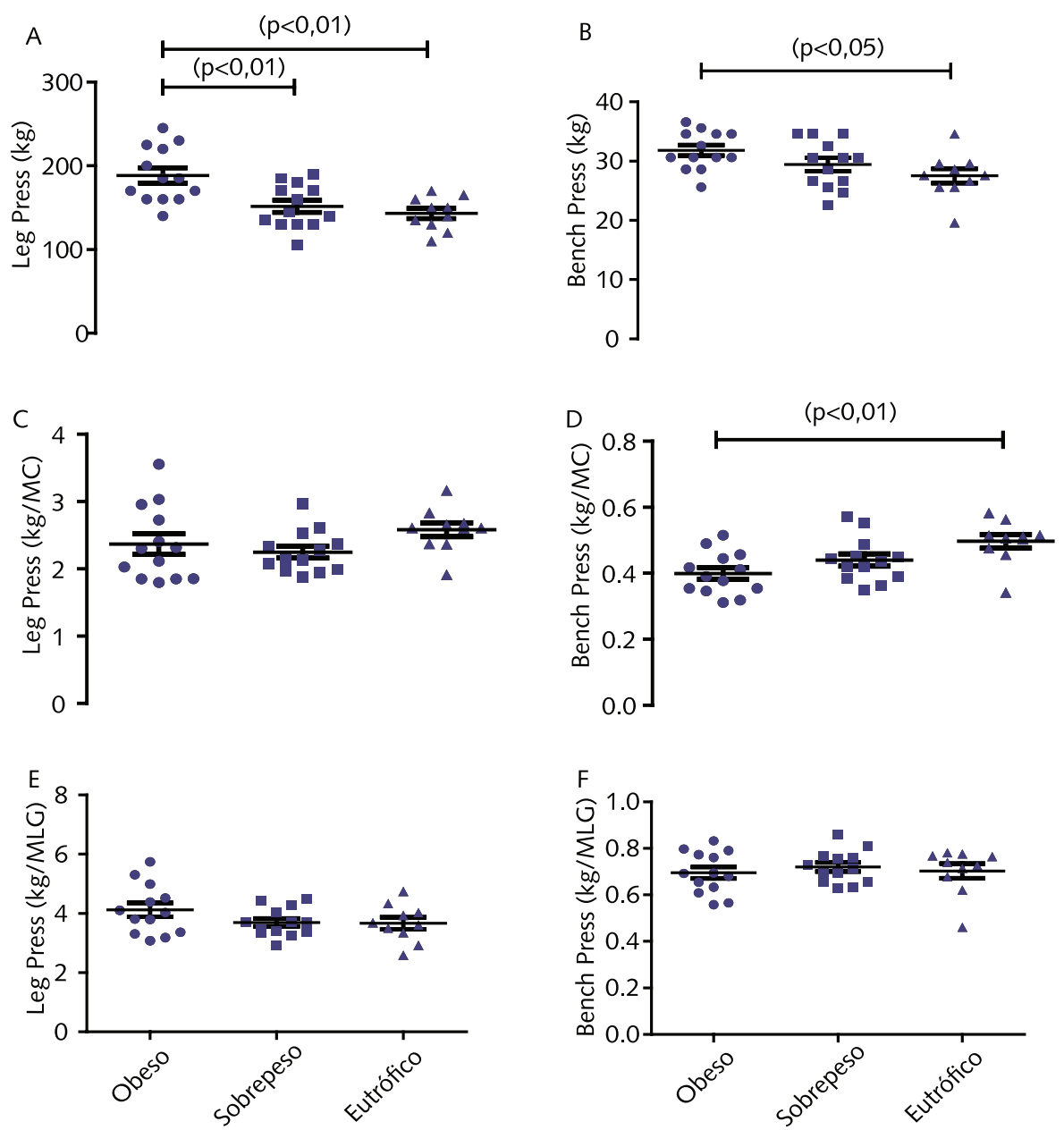

Estado Nutricional

Estado Nutriciona

Figura 1 - Carga movimentada absoluta, relativa à massa corporal $(M C)$ e relativo à massa livre de gordura (MLG) no Leg Press e no Bench Press de acordo com o estado nutricional (obeso, sobrepeso e eutrófico). (A) Leg Press (kg); (B) Bench Press (kg); (C) Leg Press (kg/MC); (D) Bench Press (kg/ $\mathrm{MC}) ;(\mathrm{E})$ Leg Press (Kg/MLG); (F) Bench Press (kg/MLG).

Tabela 1 - Características gerais dos grupos (média \pm desvio padrão).

\begin{tabular}{lccccc}
\hline \multicolumn{7}{c}{ Estado Nutricional } \\
\hline Variáveis & $\begin{array}{c}\text { Obeso } \\
(\mathrm{n}=13)\end{array}$ & $\begin{array}{c}\text { Sobrepeso } \\
(\mathrm{n}=13)\end{array}$ & $\begin{array}{c}\text { Eutrófico } \\
(\mathrm{n}=10)\end{array}$ & $\mathrm{F}$ & $\mathrm{p}$ \\
\hline Idade (anos) & $15,2 \pm 1,14$ & $14,4 \pm 0,87$ & $15,4 \pm 0,72$ & 2,50 & 0,10 \\
\hline Estágios de Tanner 4/5 (n) & $3 / 10$ & $3 / 10$ & $2 / 8$ & $0,03^{\#}$ & $=0,98$ \\
\hline MC $(\mathrm{kg})$ & $80,9 \pm 11,07^{\mathrm{a}^{* *}}$ & $67,5 \pm 8,02^{\mathrm{b}^{* *}}$ & $55,2 \pm 3,89^{\mathrm{c}^{* * *}}$ & 26,57 & $<0,0001$ \\
\hline Estatura $(\mathrm{m})$ & $1,62 \pm 0,06$ & $1,60 \pm 0,07$ & $1,64 \pm 0,04$ & 1,27 & $=0,29$ \\
\hline IMC $\left(\mathrm{kg} / \mathrm{m}^{2}\right)$ & $30,6 \pm 2,98^{\mathrm{a}^{* * *}}$ & $26,4 \pm 1,69^{\mathrm{b}^{* * *}}$ & $20,6 \pm 1,53^{\mathrm{c}^{* * *}}$ & 63,10 & $<0,0001$ \\
\hline CC $(\mathrm{cm})$ & $84,3 \pm 7,72$ & $79,4 \pm 7,63^{\mathrm{b}^{* *}}$ & $67,2 \pm 3,98^{\mathrm{c}^{* * *}}$ & 15,28 & $<0,0001$ \\
\hline MG (\%) & $42,7 \pm 3,69$ & $39,1 \pm 4,76^{\mathrm{b}^{* * *}}$ & $29,0 \pm 5,37^{\mathrm{c}^{* * *}}$ & 19,32 & $<0,0001$ \\
\hline MG (kg) & $34,9 \pm 7,43^{\mathrm{a}^{* *}}$ & $26,6 \pm 5,63^{\mathrm{b}^{* * *}}$ & $16,1 \pm 3,25^{\mathrm{c}^{* * *}}$ & 28,19 & $<0,0001$ \\
\hline MLG (\%) & $57,3 \pm 3,69$ & $60,9 \pm 4,76^{\mathrm{b}^{* * *}}$ & $70,9 \pm 5,37^{\mathrm{c}^{* * *}}$ & 19,32 & $<0,0001$ \\
\hline MLG (kg) & $46,1 \pm 4,28^{\mathrm{a}^{* *}}$ & $40,9 \pm 3,76$ & $39,3 \pm 3,77^{* *}$ & 8,59 & $<0,001$ \\
\hline
\end{tabular}

$M C=$ massa corporal; $I M C=$ =índice de massa corporal; $C C=$ ircunferência da cintura; $M G=$ massa gor-

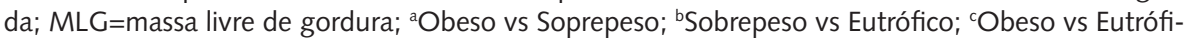
co; ${ }^{* *} \mathrm{p}<0,01 ;{ }^{* * *} \mathrm{p}<0,001$; \# comparação entre proporções por Qui-Quadrado $\left(\chi^{2}\right)$. 
Tabela 2 - Matriz de valores dos coeficientes de correlação de Pearson entre a carga movimentada nos membros inferiores e superiores (Leg Press e Bench Press) e as variáveis antropométricas e da composição corporal de obesas e não-obesas $(n=36)$.

\begin{tabular}{lccccccc}
\hline & Leg Press & $\begin{array}{c}\text { Bench } \\
\text { Press }\end{array}$ & Idade & IMC & $\%$ GC & MG & MLG \\
\hline Leg Press & 1,00 & $0,60^{* *}$ & 0,16 & $0,66^{* *}$ & $0,48^{* *}$ & $0,58^{* *}$ & $0,54^{* *}$ \\
\hline Bench Press & & 1,00 & 0,12 & $0,54^{* *}$ & 0,29 & $0,43^{* *}$ & $0,57^{* *}$ \\
\hline Idade & & 1,00 & $-0,04$ & $-0,21$ & $-0,01$ & $0,34^{*}$ \\
\hline IMC & & & 1,00 & $0,84^{* *}$ & $0,93^{* *}$ & $0,69^{* *}$ \\
\hline \%GC & & & & 1,00 & $0,93^{* *}$ & $0,37^{*}$ \\
\hline MG & & & & 1,00 & $0,67^{* *}$ \\
\hline MLG & & & & & 1,00 \\
\hline
\end{tabular}

IMC=índice de massa corporal; \%GC=percentual de gordura corporal; $M G=$ massa gorda (em kg); $M L G=$ massa livre de gordura (em kg); ${ }^{*} p<0,05 ;{ }^{*} p<0,01 ; N S=$ não significante.

ficativamente correlacionados com a carga movimentada em membros inferiores $(r=0,66, r=0,58$ e $r=0,54)$ e superiores $(r=0,54, r=0,43$ e $r=0,57)$, respectivamente. $\mathrm{O} \% \mathrm{GC}$ foi direta e significantemente correlacionado apenas com a carga movimentada nos membros inferiores $(r=0,48)$.

O teste de regressão linear múltipla mostrou que o IMC explicou 44\% ( $\mathrm{r}^{2}$ ajustado $=0,44)$ da variação da carga movimentada nos membros inferiores $(\beta=4,81$, $\mathrm{p}<0,01)$ e a MLG explicou $32 \%\left(\mathrm{r}^{2}\right.$ ajustado=0,32) da variação da carga movimentada nos membros superiores $(\beta=0,52, \mathrm{p}<0,05)$.

\section{DISCUSSÃO}

O objetivo do presente estudo foi comparar a força muscular de membros inferiores e superiores entre adolescentes obesas e não-obesas e verificar a associação da força muscular com as variáveis antropométricas e da composição corporal. $\mathrm{Na}$ presente amostra, os resultados demonstraram que adolescentes obesas têm maior força muscular absoluta tanto nos membros inferiores como nos membros superiores que às não-obesas. Estes achados corroboram com estudos anteriores que verificaram maiores valores de força muscular nos membros inferiores em adolescentes obesos ${ }^{11-14,16-19}$ e nos membros superiores em mulheres obesas ${ }^{15}$.

O excesso de massa corporal representa carga adicional na estrutura musculoesquelética de indivíduos obesos, levando a adaptações favoráveis na massa muscu$1 \mathrm{ar}^{24-26}$, massa óssea ${ }^{24-26}$ e na força muscular ${ }^{11-19}$. Além disto, os obesos apresentam perfil metabólico alterado em comparação aos não-obesos, com maiores valores de insulina basal, a qual pode levar a estado anabólico sistêmico ${ }^{27}$. A combinação deste ambiente anabólico e a sobrecarga adicional podem ser os responsáveis pela maior massa muscular e óssea, bem como os maiores níveis de força verificada em obesos quando comparados aos não-obesos.

A MLG tem sido considerada a principal responsável pelos maiores níveis de força muscular em obesos ${ }^{5}$. No presente estudo, as adolescentes obesas apresentaram maiores valores de MLG em comparação as não-obesas. Adicionalmente, a MLG apresentou associação direta e significativa com força muscular absoluta dos membros superiores, explicando $32 \%$ da variação na força. Por outro lado, nos membros inferiores foi o IMC a variável que melhor explicou a variação da força muscular absoluta (44\%). Este achado sugere que as adaptações neuromusculares nos membros inferiores podem estar relacionadas a adaptações neurais acarretadas pela sobrecarga mecânica gerada pelo peso adicional, além das adaptações hipertró- 
ficas da musculatura evidenciada em obesos ${ }^{26}$. A presença de maior estado de ativação neural nos membros inferiores do que nos superiores entre obesos e não-obesos e a relação com a carga corporal adicional necessita ser melhor investigada ${ }^{12}$.

A magnitude da diferença da força muscular absoluta entre obesas e não-obesas foi maior nos membros inferiores $(+25,5 \%)$ do que nos membros superiores $(+15,5 \%)$. Isto indica que os membros inferiores, por receberem maior influência do peso corporal nas atividades diárias (como andar, subir escadas, sentar e levantar, entre outras) apresentam maiores adaptações neuromusculares do que os membros superiores em obesos. Estas diferenças também foram evidenciadas por Vandewalle et al. ${ }^{26}$ na densidade volumétrica e na geometria óssea por tomografia computadorizada da tíbia e do rádio de adolescentes obesos belgas.

O nível de atividade física pode influenciar os níveis de força muscular, entretanto, a influência desta variável na força muscular em obesos não tem sido investigada ${ }^{11-18}$. Recentemente, Tsiros et al. ${ }^{19}$ compararam o torque máximo de extensores de joelho entre adolescentes obesos e não-obesos australianos e verificaram a influência dos níveis de atividade física, medidos por acelerômetro. Os obesos apresentaram menores níveis de atividade física que os não-obesos e houve relação inversa entre o torque máximo de joelho e níveis de atividade física. Entretanto, a variação nos níveis de atividade física não conseguiu explicar a variação na força muscular dessa população. Estes achados sugerem que a maior força muscular nos membros inferiores encontrada em obesos, pode estar mais relacionada à carga adicional promovida pelo excesso de peso durante as atividades habituais do que a quantidade destas atividades. No presente estudo, não foi medido o nível de atividade física, no entanto, nenhuma adolescente tinha histórico de atividade física maior que 200 minutos por semana, considerado abaixo da recomendação mínima de $60 \mathrm{~min} /$ dia (300 min/semana) proposta para crianças e adolescentes $^{28}$. Tendo em vista que obesos são menos ativos que os não-obesos, conforme reportado em estudos anteriores ${ }^{19,29-30}$, nossos resultados suportam a ideia de que a carga adicional da maior massa corporal seja a principal responsável pela maior capacidade de movimentar carga de obesos.

O maior tempo de exposição à obesidade pode exercer efeito negativo no tecido muscular e, consequentemente, na força muscular em adultos. Estudos recentes tem associado a obesidade com perda de massa muscular, fato este conhecido como obesidade sarcopênica ${ }^{31-32}$. O tecido adiposo tem sido considerado como glândula endócrina produtora de inúmeras substâncias inflamatórias. $\mathrm{O}$ excesso de gordura corporal, particularmente a visceral, pode levar a estado inflamatório crônico de baixo grau, com aumento de substâncias como interleucina-6, fator de necrose tumoral alfa e proteína $\mathrm{C}$ reativa ${ }^{33}$. $\mathrm{O}$ aumento destas substâncias representa estímulo catabólico ao longo do tempo. A estimulação do catabolismo na ausência de ambiente anabólico (redução das atividades físicas e dieta inadequada) pode contribuir para a perda de massa muscular e da força $a^{31}$. Neste estudo, a obesidade pareceu ter efeito positivo na força muscular absoluta, tendo em vista os maiores valores de força muscular tanto nos membros inferiores como nos membros superiores. No entanto, quando a força muscular foi expressa em relação à MLG, não houve diferenças entre obesas e não-obesas, sugerindo possível prejuízo na função neuromuscular em obesos, visto que as adolescentes obesas apresentaram maiores valores de MLG e níveis de força do que as não-obesas.

A grande novidade deste estudo é que a obesidade afetou a força muscular absoluta, estimada por teste de $1 \mathrm{RM}$, tanto dos membros inferiores como dos membros superiores nas adolescentes da amostra, sendo a magnitude deste efeito 
maior nos membros inferiores do que nos superiores. Os achados do presente estudo podem contribuir para melhor compreensão do comportamento da força muscular em adolescentes obesas, considerando que o treinamento de força tem sido incluído como alternativa de exercício físico para o tratamento da obesidade, o qual poderia ganhar mais espaço na elaboração de programas de treinamento físico para esta população.

O presente estudo apresenta algumas limitações que devem ser mencionadas. Primeiro, caracteriza-se como delineamento transversal, por isso não podemos estabelecer relação de causa e efeito entre a obesidade e a força muscular. Segundo, o número de sujeitos foi pequeno $(\mathrm{n}=36)$, comprometendo a generalização dos resultados para a população estudada. Terceiro, utilizamos o teste de 1RM para estimar a força dinâmica máxima e a composição corporal pela impedância bioelétrica, que não são considerados gold-standard para avaliação dessas variáveis. Por outro lado, a utilização desses métodos não invalidam nossos achados, visto que esses métodos apresentam boa concordância com DEXA e o dinamômetro isocinético, considerados gold-standard na avaliação da massa livre de gordura e da força muscular, respectivamente, além do fácil acesso e aplicabilidade pelos profissionais da área da saúde. Adicionalmente, a análise de regressão apresentada no presente estudo não foi controlada para possíveis variáveis de confusão como idade e estágio da maturação sexual e que, portanto, as associações apresentadas no presente estudo devem ser interpretadas com cautela. Entretanto, todos os indivíduos foram classificados no estágio 4 ou 5 de Tanner, considerados estágios finais da maturação sexual os quais sofrem menor influência na composição corporal e no desempenho físico, especialmente no sexo feminino.

Em resumo, o presente estudo demonstrou que a obesidade afetou positivamente a força muscular absoluta tanto dos membros inferiores como dos membros superiores das adolescentes da amostra estudada e que a magnitude do efeito é maior nos membros inferiores. Além disto, o IMC foi a variável que melhor explicou a variação da força muscular absoluta dos membros inferiores em adolescentes obesas e não-obesas. Estudos futuros são necessários para verificar se as diferenças na magnitude da força muscular absoluta entre membros superiores e inferiores de adolescentes obesas estão relacionadas com variações na massa livre de gordura entre estes membros ou estão associadas a alterações funcionais acarretadas pela diferente demanda funcional.

\section{Agradecimentos}

Agradecemos ao Colégio da Polícia Militar do Estado do Paraná e ao Tenente Incare Correa de Jesus pela disponibilidade do local e da população para realização do presente estudo; a Priscilla Coutinho, médica responsável pela avaliação médica dos voluntários da pesquisa; a Profa Maria de Fátima Lopes pelo empréstimo de equipamentos para realização deste estudo e a Fundação Araucária pelo financiamento do presente projeto. $\mathrm{O}$ primeiro autor e a terceira autora são bolsistas de doutorado do CNPq e CAPES, respectivamente. A segunda e a última autora são bolsistas de produtividade do CNPq.

\section{Colaboradores}

W. A. L. participou da concepção e execução do projeto, análise e interpretação dos resultados, redação do artigo e aprovação final da versão publicada, N. L. participou da revisão crítica do conteúdo intelectual e aprovação final da versão publicada, L. R. S. participou da análise e interpretação dos resultados, redação do arti- 
go, revisão crítica do conteúdo intelectual e aprovação final da versão publicada, F. B. M. J. participou da análise e interpretação dos resultados e redação do artigo e aprovação final da versão publicada, C. L. M. C. participou da análise e interpretação dos resultados, redação do artigo e aprovação final da versão publicada, C. T. A. participou da interpretação dos resultados, redação do artigo e aprovação final da versão publicada e C. R. C. participou da revisão crítica do conteúdo intelectual e aprovação final da versão publicada.

\section{REFERÊNCIAS}

1. Odgen SL, Carrol MD, Kit BK, Flegal KM. Prevalence of obesity and trends in body mass index among US children and adolescents. 1999-2010. JAMA 2012;307:483-490.

2. Gupta N, Goel K, Shah P, Misra A. Childhood obesity in developing countries: epidemiology, determinants, and prevention. Endocr Rev 2012; 33:48-70.

3. Kiess W, Galler A, Reich A, Müller G, Kapellen T, Deutscher J. Clinical aspects of obesity in childhood and adolescence. Obes Rev 2001;2:29-36.

4. Kodama S, Saito K, Tanaka, S, Maki M, Yachi Y, Asumi M, et al. Cardiorespiratory fitness as a quantitative preditor of all-cause mortality and cardiovascular events in healthy men and woman: a meta-analysis. JAMA 2009;301:2024-2035.

5. Tsiros MD, Coates AM, Howe PR, Grimshaw PN, Buckley JD. Obesity: the new childhood disability? Obes Rev. 2011;12(1):26-36.

6. Kraemer WJ, Adams K, Cafarelli E, Dudley GA, Dooly C, Feigenbaum MS, et al. American College of Sports Medicine position stand. Progression models in resistance training for healthy adults. Med Sci Sports Exerc 2002;34:364-380.

7. Artero EG, Lee D, Lavie CJ, España-Romero V, Sui X, Church TS, et al. Effects of muscular strength on cardiovascular risk factors and prognosis. J Cardiopulm Rehabil 2012;32:351-358.

8. Artero EG, Ruiz JR, Ortega FB, España-Romero V, Vicente-Rodríguez G, Molnar D, et al. Muscular and cardiorespiratory fitness are independently associated with metabolic risk in adolescents: the HELENA study. Pediatr Diabetes 2011;12:704-712.

9. Benson AC, Torode ME, Singh MA. Muscular strength and cardiorespiratory fitness is associated with higher insulin sensitivity in children and adolescents. Int J Pediatr Obes 2006;1:222-231.

10. Jiménez-Pavón D, Ortega FB, Valtueña J, Castro-Piñero J, Gómez-Martínez S, Zaccaria $\mathrm{M}$ et al. Muscular strength and markers of insulin resistance in European adolescents: the HELENA study. Eur J Appl Physiol 2012;112:2455-2465.

11. Blimkie CJ, Ebbesen B, MacDougall D, Bar-Or O, Sale D. Voluntary and electrically evoked strength characteristics of obese and nonobese preadolescents boys. Hum Biol 1989; 61(4):515-532.

12. Blimkie CJ, Sale DG, Bar-Or. Voluntary strength, evoked twitch contractile properties and motor unit activation of knee extensors in obese and non-obese adolescent males. Eur J Appl Physiol Occup Physiol 1990; 61(3-4): 313-318.

13. Maffiuletti NA, Jubeau M, Munzinger U, Bizzini M, Agosti F, De Col A, Lafortuna CL, Sartorio A. Differences in quadríceps muscle strength and fatigue between lean and obese subjects. Eur J Appl Physiol 2007;101:51-59.

14. Maffiuletti NA,Jubeau M,AgostiF,De ColA,Sartorio A.Quadriceps muscle function characteristics in severely obese and nonobese adolescents. Eur J Appl Physiol 2008;103(4):481-484.

15. Zoeller RF, Ryan, ED, Gordish-Dressman H, Price TB, Seip RL, Angelopoulos TJ, et al. Allometric scaling of isometric biceps strength in adult females and the effect of body mass index. Eur J Appl Physiol 2008;104:701-10.

16. Lazzer S, Pozzo R, Rejc E, Antonutto G, Francescato MP. Maximal explosive muscle power in obese and non-obese prepubertal children. Clin Physiol Funct Imaging 2009;29:224-228.

17. Rauch R, Veilleux LN, Rauch F, Bock D, Welisch E, Filler G, et al. Muscle force and power in obese and overweight children. J Musculoskelet Neuronal Interact 2012;12(2):80-83.

18. Abdelmoula A, Martin V, Bouchant A, Walrand S, Lavet C, Taillardat M, et al. Knee extension strength in obese and nonobese male adolescents. Appl Physiol Nutr Metab 2012 Apr;37(2):269-75. 
19. Tsiros DM, Coates AM, Howe PRC, Grimshaw PN, Walkley J, Shield A, et al. Knee extensor strength differences in obese and healthy-weight 10-13 year olds. Eur J Appl Physiol 2013;113(6):1415-1422.

20. World Health Organization. WHO child growth standards: length/height-for-age, weightfor-age, weight-for-length, weight-for height and body mass index-for-age: methods and development. Geneva: World Health Organization; 2006.

21. Houtkooper, LB, Lohman TG, Roche, AF, Van Loan, M. Bioelectrical impedance estimation of fat-free body mass in children and youth: a cross-validation study.J Appl Physiol 1992;72:366-373.

22. Tanner JM, Normal growth and techniques of growth assessment, Clin Endocrinol Metab 1986, 15: 411-45.

23. Brown LE, Weir JP. ASEP Procedures recommendation I: accurate assessment of muscular strength and power. J Exerc Physiol Online 2001;4:1-21.

24. Wetzsteon RJ, Petit MA, Macdonald HM, Hughes JM, Beck TJ, McKay HA. Bone Structure and Volumetric BMD in Overweight Children: A Longitudinal Study. J Bone Miner Res 2008;23:1946-53.

25. Gracia-Marco L, Ortega FB, Jiménez-Pavón D, Rodríguez G, Castillo MJ, Vicente-Rodríguez G. Adiposity and bone health in Spanish adolescents. The HELENA study. Osteoporos Int 2012; 23:937-47.

26. Vanderwalle S, Taes Y, Van Helvoirt M, Debode P, Herregods N, Ernst C, et al. Bone size and bone strength are increased in obese male adolescents. J Clin Endocrinol Metab 2013;98:30193028.

27. Kern PA, Simsolo RB, Fournier M. Effect of weight loss on muscle fiber type, fiber size, capillarity, and succinate desidrogenase activity in humans. J Clin Endocrinol Metab 1999;84(11):4185-90.

28. World Health Organization. Global Recommendations on Physical Activity for Health. Geneva: World Health Organization, 2010

29. Farr JN, Van Loan MD, Lohman TG, Going SB. Lower physical activity is associated with skeletal muscle fat content in girls. Med Sci Sports Exerc 2012;44(7):1375-1381.

30. Brito AKA, Silva Junior FL, Coelho LS, França NM. Nível de atividade física e correlação com o índice de massa corporal e percentual de gordura em adolescentes escolares da cidade de Teresina-PI. Rev Bras Ativ Fis e Saúde 2012;17(3):212-216.

31. Benton MJ, Whyte MD, Dyal BW. Sarcopenic obesity: strategies for management. Am J Nurs 2011;111:38-44.

32. Beyer I, Mets T, Bautmans I. Chronic low-grade inflammation and age-related sarcopenia. Curr Opin Clin Nutr Metab Care 2012;15:12-22.

33. Gleeson M, Bishop NC, Stendel DJ, Lindley MR, Mastana SS, Nimmo MA. The antiinflammatory effects of exercise: mechanisms and implications for the prevention and treatment of disease. Nat Rev Immunol 2011;11: 607-615.

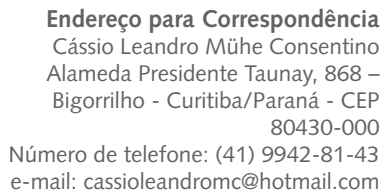

